\title{
Relação entre níveis de compreensão e estratégias de leitura utilizadas por surdos sinalizadores em um programa terapêutico
}

\author{
Relationship between comprehension levels and reading \\ strategies used in a therapeutic program by deaf individuals \\ who communicate through sign language
}

\author{
Maria Sílvia Cárnio $^{1}$, Eleonora Silva Csipai $^{2}$, Maria Inês Vieira Couto ${ }^{3}$
}

\begin{abstract}
RESUMO
Objetivo: Determinar as mudanças referentes às estratégias de aprendizagem nos níveis de compreensão de leitura durante um programa de intervenção fonoaudiológica direcionado aos surdos sinalizadores. Métodos: Participaram cinco estudantes surdos, com idades entre dez anos e 15 anos, cuja língua preferencial para comunicação era a Língua Brasileira de Sinais. Todos frequentaram um programa de intervenção fonoaudiológica fundamentada na técnica de scaffolding (andaime). As variáveis consistiram dos desempenhos das avaliações individuais do nível de compreensão de leitura e das estratégias utilizadas para esta compreensão, antes de iniciar o programa e ao término do mesmo. Resultados: Ao término do programa de intervenção, mais estudantes surdos utilizaram estratégias de elaboração $(\mathrm{p}=0,197)$ e menos estudantes utilizaram estratégias de monitoramento $(\mathrm{p}=0,197)$. Houve mudança significativa quanto à pontuação do nível de decodificação $(\mathrm{p}=0,109)$ e tendência à significância no nível de compreensão literal $(\mathrm{p}=0,197)$, com aumento da pontuação. Verificou-se significância somente entre a utilização da estratégia de elaboração e os dois momentos da avaliação (inicial e final) $(\mathrm{p}=0,059)$. Conclusão: O programa de intervenção baseado na técnica de scaffolding propiciou que estudantes surdos sinalizadores utilizassem mais estratégias de elaboração e menos de monitoramento; consequentemente, uma tendência para a passagem do nível de decodificação para o nível de compreensão literal do texto.
\end{abstract}

Descritores: Surdez; Compreensão; Leitura; Linguagem de sinais; Eficiência organizacional

\section{INTRODUÇÃO}

A leitura de um texto consiste nas habilidades de decodificação, compreensão e interpretação ${ }^{(1)}$.

A compreensão é uma tarefa que inclui diferentes processos cognitivos, como memória e inferência; diferentes níveis de complexidade, denominados de baixo nível (identificação de

Trabalho realizado no Laboratório de Leitura e Escrita do Departamento de Fisioterapia, Fonoaudiologia e Terapia Ocupacional da Faculdade de Medicina da Universidade de São Paulo - USP - São Paulo (SP), Brasil.

(1) Doutora, Professora Associada do Curso de Fonoaudiologia do Departamento de Fisioterapia, Fonoaudiologia e Terapia Ocupacional da Faculdade de Medicina da Universidade de São Paulo - USP - São Paulo (SP), Brasil.

(2) Fonoaudióloga graduada pelo Curso de Fonoaudiologia da Faculdade de Medicina da Universidade de São Paulo - USP - São Paulo (SP), Brasil.

(3) Doutora, Fonoaudióloga do Departamento de Fisioterapia, Fonoaudiologia e Terapia Ocupacional da Faculdade de Medicina da Universidade de São Paulo - USP - São Paulo (SP), Brasil.

Endereço para correspondência: Maria Silvia Cárnio. R. Cipotânea, 51, Cidade Universitária, São Paulo (SP), Brasil, CEP: 05360-160. E-mail: mscarnio@usp.br

Recebido em: 4/6/2009; Aceito em: 30/10/2009 palavras e letras) e alto nível (elaboração de inferências) ${ }^{(2)}$; e, diferentes fatores, tais como vocabulário adquirido, a fluência da e motivação pela leitura ${ }^{(3-5)}$. Contudo, a compreensão de leitura sofre também a interferência das sequências de técnicas ou de atividades que o leitor utiliza com o propósito de facilitar a aquisição, o armazenamento e/ou a utilização da informação,denominadas de estratégias de aprendizagem (ensaio, elaboração, organização, monitoramento e afetivas) ${ }^{(6)}$. Tais técnicas possibilitam a reconstrução do significado do texto com base na integração de informações e inferências.

A literatura citada refere-se aos estudantes ouvintes. Sabese que estudantes surdos, cuja primeira língua é a de sinais, passam por processos diferentes para a aquisição e aprimoramento da segunda língua, na modalidade escrita ${ }^{(7,8)}$. Estudos evidenciam que durante a leitura de um texto, os surdos sinalizadores atêm-se mais às ilustrações do que os leitores ouvintes $^{(9)}$; utilizam a datilologia ${ }^{(10)}$, o apontamento do texto, apoio articulatório e gestos durante a leitura ${ }^{(11)}$. Portanto, ao se elaborar um programa de intervenção fonoaudiológico, para que os estudantes surdos sinalizadores aprimorem a compre- 
ensão da leitura de texto, é necessário certificar a qualidade do serviço prestado pelo programa de intervenção.

Visto que os fatores inerentes à leitura de um texto e os recursos externos acessíveis influenciam na compreensão da leitura de estudantes surdos, levanta-se a hipótese de que existe uma relação positiva entre estratégias de aprendizagem e o nível de compreensão de leitura: quanto mais estratégias o estudante surdo sinalizador utilizar, melhor será o nível de compreensão. O presente estudo teve como objetivo determinar as mudanças referentes às estratégias de aprendizagem nos níveis de compreensão de leitura durante um programa de intervenção fonoaudiológica direcionado aos surdos sinalizadores.

\section{MÉTODOS}

Essa pesquisa clínica, longitudinal e retrospectiva, foi aprovada pelo Comitê de Ética para Análise de Projetos de Pesquisa (CAPPesq) da Faculdade de Medicina da Universidade de São Paulo (FMUSP) sob o n0607/07. Os responsáveis pelos sujeitos assinaram um Termo de Consentimento Livre e Esclarecido.

A coleta dos dados foi realizada no Laboratório de Investigação Fonoaudiológica de Leitura e Escrita (LIFLE) do Curso de Fonoaudiologia da Faculdade de Medicina da USP.

\section{Participantes}

Para atingir os objetivos propostos, foi necessário utilizar um estudo longitudinal. Neste percurso, houve variação do número de sujeitos: no início do programa havia 12 sujeitos; sete foram retirados do estudo por não cumprirem o número de sessões estabelecidas por diversos motivos, tais como, mudança do horário escolar, não implicação dos pais e indisponibilidade de tempo por inclusão no mercado de trabalho.

Participaram cinco estudantes com perda auditiva neurossensorial de grau profundo nas duas orelhas; dois estudantes do gênero feminino e três do masculino; filhos de pais ouvintes e da classe econômica D, conforme a classificação da Associação Brasileira de Empresas de Pesquisa (2008)(12). A idade variou de dez anos a 15 anos (média de 12 anos e oito meses; $\mathrm{DP}=2,3$ ). Todos frequentavam escola pública especial e estavam no ensino fundamental (dois na $3^{\mathrm{a}}$ série, dois na $4^{\mathrm{a}}$ série e um na $5^{\mathrm{a}}$ série). A língua preferencial para comunicação dos participantes era a Língua Brasileira de Sinais (Libras). Todos os sujeitos já frequentavam terapia fonoaudiológica no LIFLE, encaminhados para este serviço com queixas dos professores e/ou familiares em relação à aquisição e desenvolvimento do português na modalidade escrita.Contudo, não apresentavam alterações cognitivas, neurológicas e de aprendizagem da língua de sinais (Libras). O laboratório desenvolve o serviço fundamentado na Filosofia de Educação Bilíngue ${ }^{(13)}$ : aquisição e aprimoramento da Libras e da Língua Portuguesa na modalidade escrita.

\section{Equipamentos e materiais}

Os materiais utilizados nesta pesquisa foram cinco DVDs com as gravações das avaliações dos sujeitos participantes da pesquisa e dois protocolos. O primeiro, Protocolo de Avaliação de um Programa Terapêutico de Habilidades Instrumentais de Leitura*, foi utilizado com o objetivo de avaliar o nível de compreensão de leitura do estudante (decodificação, compreensão literal, compreensão independente e leitura crítica). O segundo, Protocolo de Estratégias de Leitura**, foi utilizado para verificar quais as estratégias o sujeito usa durante a leitura de um texto.

Para assistir os DVDs foi utilizado o leitor de DVD e um monitor.

\section{Programa de intervenção}

Entre as avaliações inicial e final, os sujeitos participaram de um programa de intervenção com objetivo de aprimorar a compreensão de leitura, por meio da técnica de scaffolding (técnica de estruturação ou andaime). Esta técnica foi desenvolvida por Hoffman em $1997^{(14)}$, com crianças pré-escolares com atraso no desenvolvimento fonológico. Consiste na recontagem oral de livros de histórias na qual adulto e criança lêem simultaneamente uma história e a seguir ambos recontam-na e constroem uma sequência dos fatos, num material específico (folha de sulfite ou computador, por exemplo). Os resultados desta técnica evidenciaram a sua eficácia para o desenvolvimento fonológico e linguístico ${ }^{(15,16)}$.

A Figura 1 exemplifica o esquema da técnica de scaffolding, quando utilizada a história "Procurando Nemo".

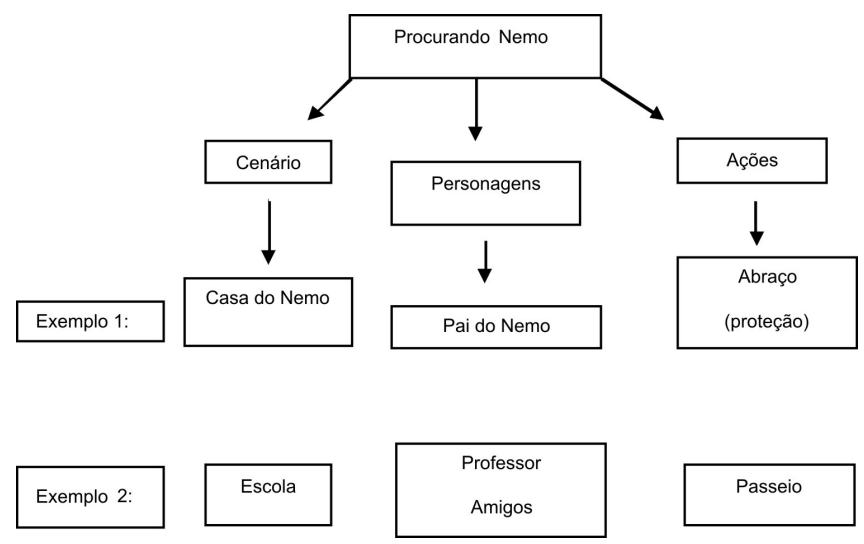

Figura 1. Esquema da técnica de scaffolding, quando utilizada a história "Procurando Nemo"

A recontagem apresenta como objetivos: organização temporal do discurso, topicalização, aprimorar as habilidades de leitura, escrita, relação causa/efeito, aspectos linguísticos e estratégias de planejamento ${ }^{(14,17,18)}$.

O programa realizado no Laboratório, com base nesta

* Cárnio MS, Zacharias L. Protocolo de habilidades instrumentais de leitura.São Paulo: Departamento de Fisioterapia, Fonoaudiologia e Terapia Ocupacional da Faculdade de Medicina da Universidade de São Paulo; 2006. [Protocolo não publicado]

** Cárnio MS, Silva EC. Protocolo de estratégias de leitura. São Paulo: Departamento de Fisioterapia, Fonoaudiologia e Terapia Ocupacional da Faculdade de Medicina da Universidade de São Paulo; 2007. Adaptado de Boruchovitch et al. 2006 ${ }^{(6)}$. [Protocolo não publicado]. 
técnica foi realizado em19 sessões, uma vez por semana, de 50 minutos cada, durante seis meses, com interrupção por três semanas, no período de férias escolares.

Os procedimentos realizados neste programa de intervenção consistiam: (1) da leitura compartilhada entre terapeuta e o sujeito de um trecho de um livro clássico de história da literatura infanto-juvenil,ou seja, cada um lia uma parte do trecho escolhido. Foram trabalhados trechos de três livros selecionados conforme o grau crescente de complexidade, da coleção "Grandes Clássicos Disney" (Editora Edelbra): "Tarzan, o rei da Selva", "Procurando Nemo" e "Nem que a Vaca Tussa";(2) da recontagem em Libras pelos estudantes surdos do trecho lido; (3) da construção de um "andaime" (esquema), a partir do relato dos sujeitos e escrito pela fonoaudióloga numa folha de sulfite (técnica scaffolding).

\section{Procedimentos das avaliações inicial e final}

A avaliação das habilidades de compreensão de leitura foi realizada pela segunda autora,capacitada para a aplicação do método. Compreendeu as seguintes etapas: (a) assistir as gravações das avaliações dos sujeitos; (b) transcrever a recontagem da história, com o auxílio de um intérprete de Libras; e (c) preencher os dois protocolos. Para a avaliação dos níveis de compreensão de leitura, que incluem quatro etapas e cada uma apresenta as habilidades específicas, foram utilizados os seguintes critérios ${ }^{(19)}$ :

1) Nível de decodificação: com seis habilidades, no qual o leitor reconhece letras; reconhece palavras; discrimina letras; realiza leitura de palavras; realiza leitura de frases; e de orações simples. Para cada habilidade observada, marcaram-se dois pontos, totalizando 12 pontos.

2) Nível de compreensão literal: com cinco habilidades, no qual o leitor capta o sentido de orações; relata o que leu; retém fatos ou detalhes específicos; responde questões em relação ao conteúdo explícito do texto e realiza paráfrase. Para cada habilidade observada, marcaram-se quatro pontos, totalizando 20 pontos.

3) Nível de compreensão independente: com seis habilidades, no qual o leitor identifica as idéias principais do texto; estabelece relações entre idéias principais e secundárias, faz relações entre causa e efeito, formula previsões, hipóteses e inferências, realiza analogias e extrai conclusões. Para cada habilidade observada, marcaram-se seis pontos, totalizando 36 pontos.

4) Nível de leitura crítica: com cinco habilidades, no qual o leitor faz críticas e argumenta; avalia a superficialidade/ profundidade com que o tema foi tratado pelo autor; reconhece ambiguidades, confusões e imprecisões; valoriza a pertinência ou alcance das conclusões ou generalizações; e disserta sobre o assunto. Para cada habilidade observada, marcaram-se oito pontos, totalizando 40 pontos.

Ao final, foram somadas as pontuações de cada sujeito. A pontuação final neste quesito pode variar de zero a 108 pontos.

Para avaliação das estratégias de leitura, a mesma fonoaudióloga seguiu as etapas: (a) assistir as filmagens; (b) identificar e transcrever de forma ortográfica as estratégias de leitura que cada sujeito utilizou durante a leitura, com o auxílio de um intérprete de Libras; e (c) preencher o Protocolo de Estratégias Utilizadas pelos Surdos durante a compreensão de leitura. Os dados foram analisados segundo as categorias de estratégias propostas por Boruchovitch ${ }^{(20)}$ :

1) Ensaio: consiste em o leitor repetir ativamente, tanto pela fala como pela escrita, o material a ser aprendido;

2) Elaboração: implica na realização de conexões entre o material novo a ser aprendido e o material antigo e/ou familiar, como reescrever, resumir, criar analogias e tomar notas que vão além da simples repetição;

3) Organização: refere-se à imposição de estrutura ao material a ser aprendido, ou seja a subdivisão em partes, como elaborar tópicos, criar uma hierarquia ou rede de conceitos;

4) Monitoramento: implica que o indivíduo esteja constantemente com a consciência realista do quanto ele está sendo capaz de captar e absorver o conteúdo que está sendo ensinado, como tomar alguma providência quando se percebe que não entendeu e auto questionamento para investigar se houve compreensão;

5) Afetivas: referem-se à eliminação de sentimentos desagradáveis, que não condizem com a aprendizagem, como estabelecimento e manutenção da motivação, manutenção da atenção e concentração e controle da ansiedade.

Foram considerados dois critérios de análise: o critério "não" (zero ponto), refere-se ausência do uso de estratégia; o critério "sim" (um ponto), significa que utiliza a estratégia, independente do número de vezes em que esta foi utilizada. A pontuação final neste quesito pode variar de zero a cinco pontos.

\section{Análise dos dados}

Os dados coletados foram analisados com o propósito de responder às seguintes questões: 1) Houve mudança do número de sujeitos que utilizaram as estratégias entre a avaliação inicial e final?; 2) Houve mudança na pontuação do nível de compreensão entre a avaliação inicial e final?; 3) Houve relação entre as estratégias utilizadas e o nível de compreensão?.

Para verificar se houve mudança na pontuação dos sujeitos com diferentes características, foi utilizada análise do tipo intra-sujeito, na qual se comparou os resultados entre as pontuações da avaliação inicial e avaliação final.

Posteriormente, o banco de dados e a análise estatística foram processados pelos programas SPSS V16 e Minitab 15. Para análise estatística dos dados foram utilizados os testes não paramétricos: teste de Wilcoxon, para comparar a pontuação das estratégias utilizadas para compreensão de leitura entre as duas avaliações; e, a correlação de Spearman, para medir o grau de relação entre a decodificação e as estratégias de leitura. Foi necessário utilizar um erro estatístico acima do usualmente utilizado (5\%), devido à baixa amostragem, que limita a generalização dos dados: o grau de significância utilizado neste estudo foi de $\mathrm{p}<0,15$. Os intervalos de confiança foram construídos com $95 \%$ de confiança estatística.

\section{RESULTADOS}

As mudanças nas pontuações alcançadas por cada sujeito, 
Tabela 1. Pontuação dos sujeitos em cada nível de compreensão de leitura

\begin{tabular}{|c|c|c|c|c|c|c|c|c|c|c|}
\hline \multirow[t]{2}{*}{ Níveis de compreensão de leitura } & \multicolumn{2}{|c|}{ Sujeito 1} & \multicolumn{2}{|c|}{ Sujeito 2} & \multicolumn{2}{|c|}{ Sujeito 3} & \multicolumn{2}{|c|}{ Sujeito 4} & \multicolumn{2}{|c|}{ Sujeito 5} \\
\hline & Av. inicial & Av. final & Av. inicial & Av. final & Av. inicial & Av. final & Av. inicial & Av. final & Av. inicial & Av. final \\
\hline Decodificação & 9 & 9 & 9 & 11 & 5 & 9 & 9 & 9 & 0 & 8 \\
\hline Compreensão literal & 8 & 8 & 0 & 8 & 0 & 6 & 6 & 4 & 0 & 2 \\
\hline Compreensão independente & 0 & 0 & 0 & 0 & 0 & 0 & 0 & 0 & 0 & 0 \\
\hline Leitura crítica & 0 & 0 & 0 & 0 & 0 & 0 & 0 & 0 & 0 & 0 \\
\hline Total & 17 & 17 & 9 & 19 & 5 & 15 & 15 & 13 & 0 & 10 \\
\hline
\end{tabular}

Legenda: $A v$. = avaliação

Tabela 2. Comparação da pontuação dos níveis de compreensão de leitura entre a avaliação inicial e a final

\begin{tabular}{|c|c|c|c|c|c|c|c|}
\hline Níveis de compreensão & Avaliação & Média & Mediana & DP & $\mathrm{N}$ & IC & Valor de $p$ \\
\hline \multirow{2}{*}{ Decodificação } & Inicial & 6,40 & 9 & 3,97 & 5 & 3,48 & $0,109^{*}$ \\
\hline & Final & 9,20 & 9 & 1,10 & 5 & 0,96 & \\
\hline \multirow{2}{*}{ Compreensão literal } & Inicial & 2,80 & 0 & 3,90 & 5 & 3,42 & $0,197 \#$ \\
\hline & Final & 5,60 & 6 & 2,61 & 5 & 2,29 & \\
\hline \multirow{2}{*}{ Compreensão independente } & Inicial & 0,00 & 0 & 0,00 & 5 & - & 1,000 \\
\hline & Final & 0,00 & 0 & 0,00 & 5 & - & \\
\hline \multirow{2}{*}{ Leitura crítica } & Inicial & 0,00 & 0 & 0,00 & 5 & - & 1,000 \\
\hline & Final & 0,00 & 0 & 0,00 & 5 & - & \\
\hline \multirow{2}{*}{ Total } & Inicial & 9,20 & 9 & 7,01 & 5 & 6,15 & $0,131^{*}$ \\
\hline & Final & 14,80 & 15 & 3,49 & 5 & 3,06 & \\
\hline
\end{tabular}

* Valores estatisticamente significantes $(p<0,15)$

\# Valores com tendência à significância estatística

Legenda: $\mathrm{N}=$ número; $\mathrm{DP}=$ desvio-padrão; $I \mathrm{C}$ = intervalo de confiança

nas avaliações inicial e final de compreensão de leitura, estão evidenciadas nos níveis de decodificação e compreensão literal, ou seja, os surdos sinalizadores deste estudo necessitaram de auxílio para realização das tarefas em ambas as avaliações. Os participantes não atingiram os níveis de compreensão de leitura mais elaborados. Verificou-se que a pontuação na avaliação final do S4 piorou, que a do S1 não modificou, e que as pontuações dos S2, S3 e S5 melhoraram no final do programa de intervenção (Tabela 1).

Não houve diferença estatística do número de participantes na avaliação inicial e na final que utilizaram as estratégias ensaio ( $p=1,000)$, organização $(p=0,292)$ e afetivas $(p=1,000)$. Contudo, houve tendência à significância do número de sujeitos que utilizaram as estratégias elaboração $(p=0,197)$ e monitoramento ( $\mathrm{p}=0,197)$, ou seja, ao término do programa de intervenção, mais estudantes surdos utilizaram estratégias de

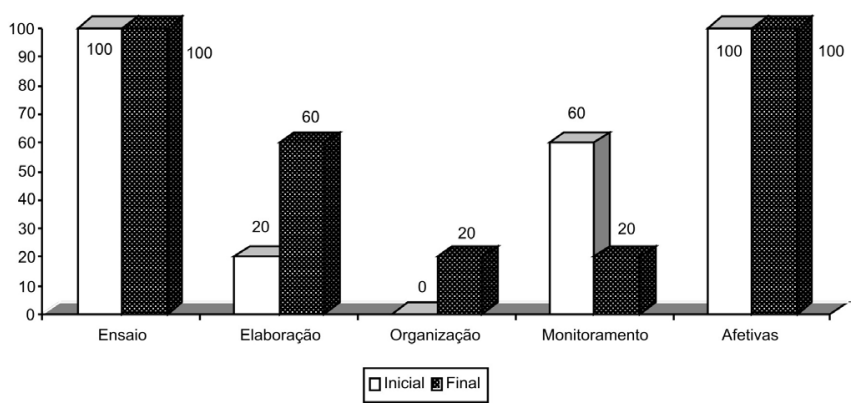

Figura 2. Distribuição dos sujeitos segundo a utilização das Estratégias de Leitura nas avaliações inicial e final elaboração (de $20 \%$ para $60 \%$ ) e menos estudantes utilizaram estratégias de monitoramento (de $60 \%$ para $20 \%$ ), conforme distribuição na Figura 2.

Referente à pontuação do nível de compreensão de leitura entre as duas situações, verificou-se diferença estatisticamente significante para o nível de decodificação $(\mathrm{p}=0,109)$ e pontuação total $(\mathrm{p}=0,131)$. Nas duas situações podemos verificar na Tabela 2 que houve aumento na pontuação. Houve tendência à significância no nível de compreensão literal $(\mathrm{p}=0,197)$, com aumento da pontuação.

De acordo com a Tabela 3, verificou-se significância somente entre a utilização da estratégia de elaboração e os dois momentos da avaliação (inicial e final) $(\mathrm{p}=0,059)$, ou seja,

Tabela 3. Relação entre o nível de decodificação de compreensão de leitura e as estratégias de leitura

\begin{tabular}{lcccc}
\hline Estratégias de leitura & & \multicolumn{3}{c}{ Decodificação } \\
\cline { 3 - 5 } & & Inicial & Final & Ambas \\
\hline Ensaio & Correlação & $0,0 \%$ & $64,5 \%$ & $31,4 \%$ \\
& Valor de p & 1,000 & 0,239 & 0,377 \\
\hline Elaboração & Correlação & $39,5 \%$ & $64,5 \%$ & $61,3 \%$ \\
& Valor de p & 0,510 & 0,239 & $0,059^{*}$ \\
\hline Monitoramento & Correlação & $-29,5 \%$ & $0,0 \%$ & $-39,1 \%$ \\
& Valor de p & 0,630 & 1,000 & 0,264 \\
\hline Afetivas & Correlação & $-39,5 \%$ & $-50,0 \%$ & $-35,4 \%$ \\
& Valor de p & 0,510 & 0,391 & 0,315 \\
\hline
\end{tabular}

*Valores estatisticamente significantes $(p<0,15)$ 
quanto maior a ocorrência do uso da estratégia de elaboração, maior a pontuação na decodificação e vice versa.

\section{DISCUSSÃO}

O presente estudo teve como objetivo principal verificar as mudanças nas estratégias de aprendizagem nos níveis de compreensão de leitura durante um programa de intervenção fonoaudiológica, fundamentado na técnica de scaffolding ${ }^{(14)}$, direcionado aos surdos sinalizadores.

Trata-se de um estudo relevante, uma vez que engloba uma população com características muito frequentes em surdos sinalizadores de classe econômica baixa, estudantes de escolas públicas especiais, que apresentam um desempenho, relativo aos conteúdos programáticos escolares, aquém do esperado para suas faixas de escolaridade ${ }^{(21)}$. Portanto, os resultados obtidos podem auxiliar outros profissionais e/ou pesquisadores na ampliação de conhecimentos sobre a compreensão de leitura de surdos sinalizadores.

A análise intra-sujeito mostrou que o $\mathrm{S} 4$ apresentou o mesmo desempenho em relação à decodificação, mas não utilizou na avaliação final as mesmas habilidades do nível de compreensão literal quando comparadas às da avaliação inicial, ou seja, utilizou parcialmente a habilidade de reter fatos ou detalhes específicos da história. O S1 não evoluiu no nível de compreensão de leitura; contudo, passou a utilizar estratégias complexas como ensaio e elaboração. Este fato indica que este sujeito provavelmente tenha um distúrbio específico de leitura e que seja necessário um programa de maior duração para que possa atingir um nível de compreensão de leitura mais elevado. Os sujeitos 2, 3 e 5 progrediram na compreensão de leitura, por meio da utilização de mais habilidades de decodificação e atingiram o nível de compreensão literal, fato que pode ser explicado pela utilização de estratégias de leitura mais sofisticadas que possibilitaram a compreensão das idéias principais explícitas no texto.

Portanto, os resultados mostraram que ao término do programa de intervenção, houve tendência de mais estudantes surdos utilizarem estratégias de elaboração e menos estudantes utilizarem estratégias de monitoramento.

A estratégia de elaboração, representada pelas atividades do estudante surdo resumir o texto e fazer conexões entre o material novo e o já conhecido, pode estar relacionada às habilidades metacognitivas ${ }^{(22)}$, trabalhadas exaustivamente na técnica de scaffolding, que promovem um exercício contínuo para a compreensão de leitura.

Houve redução significante do número de sujeitos que utilizaram a estratégia de monitoramento, na qual os estudantes surdos não necessitavam utilizar, no final do programa, recursos tais como: "recorrer ao fonoaudiólogo quando não entendiam o significado de determinadas palavras". Tal achado permite que se interprete que o programa terapêutico promoveu ampliação do vocabulário na modalidade escrita, gerando confiança e autonomia na leitura ${ }^{(10)}$.

Os resultados evidenciaram que os estudantes surdos sinalizadores não modificaram de forma significativa a utilização das estratégias ensaio e afetivas, consideradas mais elementares no processo de compreensão de leitura. No início do programa terapêutico, todos os estudantes já utilizavam a sinalização e o apoio da gravura na compreensão de leitura, corroborando os achados da literatura ${ }^{(9,22)}$. Em relação às estratégias afetivas, deve-se considerar que todos os sujeitos tiveram reações diante da atividade de leitura. Contudo, os estudantes apresentaram diferentes comportamentos durante as avaliações, tais como ansiedade e inquietação para terminar logo a atividade, explicitados verbalmente como sendo situações desagradáveis. Fato que raramente ocorreu durante o programa terapêutico, demonstrando que a motivação para ler e a descontração interferem no comportamento e na compreensão de leitura ${ }^{(4)}$.

Além disso, é importante que se enfatize o fato de que estes sujeitos inicialmente mencionavam que estavam muito habituados a serem avaliados por meio de testes de leitura de palavras e/ou de textos pequenos, reclamando do comprimento dos textos de avaliação, julgados como muito extensos.

Em relação às estratégias de organização, consideradas mais complexas, na avaliação final somente um sujeito apresentou tal estratégia. Entende-se que o programa terapêutico auxiliou-o a perceber os tópicos mais importantes do texto, enquadrando-os numa sequência temporal dos fatos. Consequentemente, a releitura facilitou a compreensão do texto corroborando achados de outros estudos ${ }^{(9,20)}$.

Outro resultado relevante foi a mudança significativa quanto à pontuação do nível de decodificação e tendência à significância no nível de compreensão literal, com aumento da pontuação. Tal fato pode ser explicado pela dinâmica da técnica de scaffolding, que possibilita o desenvolvimento de um programa terapêutico, com multiplicidade de objetivos linguísticos. Estes foram trabalhados numa situação de leitura e escrita contextualizada por meio de três livros de histórias de literatura, nos quais alguns conteúdos eram ampliados e revistos, de acordo com as necessidades dos surdos durante em 19 sessões terapêuticas.

Apesar dos resultados positivos anteriormente apresentados, o programa terapêutico não foi suficiente para possibilitar uma evolução maior na compreensão de leitura, pois nenhum dos sujeitos atingiu os níveis mais elaborados de compreensão de leitura independente e crítica. Talvez estes sujeitos precisassem de um programa mais longo para atingir graus mais elevados de compreensão de leitura ${ }^{(2,4,9)}$. Por outro lado, se estes surdos tivessem sido avaliados somente por meio de testes de leitura de palavras, todos teriam alcançado o nível de compreensão de leitura independente, não possibilitando uma avaliação mais precisa das capacidades e evoluções individuais de cada sujeito.

Verificou-se relação positiva apenas entre a estratégia de elaboração e o aumento da pontuação do nível de decodificação, uma vez que os surdos fizeram mais conexões entre os materiais lidos em uma sessão com a posterior, reescrevendo seus textos e resumindo suas idéias. Dessa forma, a decodificação de leitura tornou-se uma tarefa mais fácil e mais significativa para essa população.

Os protocolos para analisar as estratégias de compreensão de leitura e a técnica de scaffolding utilizados no presente estudo são instrumentos metodológicos que contribuíram para obtenção dos resultados benéficos para a melhora na compreensão de leitura dos participantes. O primeiro auxilia 
o fonoaudiólogo clínico a observar de forma sistemática e detalhada as habilidades e processos individuais de cada estudante durante a tarefa de leitura; e, o segundo auxilia o surdo na organização da estrutura do português escrito, na utilização de diferentes categorias gramaticais, na compreensão da sequência lógico-temporal dos fatos e na topicalização dos temas do texto escrito.

\section{CONCLUSÃO}

O programa de intervenção baseado na técnica de scaffolding propiciou que estudantes surdos sinalizadores utilizassem mais estratégias de elaboração e menos de monitoramento; consequentemente, uma tendência para a passagem do nível de decodificação para o nível de compreensão literal do texto.
Os protocolos de avaliação e a proposta terapêutica apresentados neste estudo podem contribuir tanto para a avaliação quanto para a terapia fonoaudiológica, pois permitem uma análise objetiva da decodificação e compreensão de leitura de textos, bem como do desenvolvimento da consciência metalinguística dos surdos sinalizadores no processo de compreensão de leitura textual.

\section{AGRADECIMENTOS}

À Fundação de Amparo à Pesquisa do Estado de São Paulo (FAPESP), pelo apoio financeiro concedido por meio de Auxílio à Pesquisa (processo no 2004/06247-9) e Bolsa de Iniciação Científica (processo nº 2007/55448-5).

\begin{abstract}
Purpose: To establish changes in learning strategies at reading comprehension levels during a speech-language intervention program for deaf individuals who use sign language. Methods: Five deaf students, with ages between ten and 15 years, whose preferential modality of communication was the Brazilian Sign Language, participated in the study. All subjects were participating in a speechlanguage intervention program based on the scaffolding technique. The variables were both the performance of individual assessments of reading comprehension level and strategies used for this purpose, before and after the program. Results: At the end of the intervention program, there were more students using elaboration strategies ( $\mathrm{p}=0.197)$, and less students using monitoring strategies $(\mathrm{p}=0.197)$. There was a significant change in the score of the decoding level $(\mathrm{p}=0.109)$, and a tendency to literal comprehension $(\mathrm{p}=0.197)$, with increasing scores. Significance was found only between the use of elaboration strategy and the two assessment moments (at the beginning and at the end of the program) $(\mathrm{p}=0.059)$. Conclusion: The intervention program based on scaffolding technique, enabled deaf students who communicate through sign language to use more elaboration strategies and less monitoring strategies. Therefore, there was a tendency to change from decoding level to literal reading comprehension level.
\end{abstract}

Keywords: Deafness; Comprehension; Reading; Sign language; Efficiency, organizational

\section{REFERÊNCIAS}

1. Wauters LN, van Bon WH, Tellings AE, van Leeuwe JF. In search of factors in deaf and hearing children's reading comprehension. Am Ann Deaf.2006;151(3):371-80.

2. Cain K, Bryant P, Oakhill J.Children's reading comprehension ability: concurrent prediction by working memory, verbal ability, and component skills. J Educ Psychol. 2004;96(1):31-42.

3. Giangiacomo MCPB, Navas ALGP. A influência da memória operacional nas habilidades de compreensão de leitura me escolares de $4^{\mathrm{a}}$ série. Rev Soc Bras Fonoaudiol.2008;13(1):69-74.

4. Ehren BJ. Partnerships to support reading comprehension for students with language impairment. Top Lang Disord. 2006;26(1):42-54.

5. Connor CM, Morrison FJ, Petrella JN. Effective reading comprehension instruction: examining child $\mathrm{x}$ instruction interactions. J Educ Psychol. 2004;96(4):682-98.

6. Boruchovitch E,Santos AAA, Costa ER, Neves ERC, Cruvinel M, Primi R, Guimarães SER. A construção de uma escala de estratégias de aprendizagem para alunos do ensino fundamental.Psicol Teor Pesqui. 2006;22(3):297-304.

7. Rathmann C, Mann W, Morgan G. Narrative structure and narrative development in deaf children. Deafness Educ Int. 2007;9(4):187-96.

8. Burman D, Nunes T, Evans D. Writing profiles of deaf children taught through British sign language. Deafness Educ Int. 2007;9(1):2-23.

9. Schirmer BR, Bailey J, Lockman AS. What verbal protocols reveal about the reading strategies of deaf students: a replication study. Am
Ann Deaf.2004;149(1):5-16.

10. Puente A, Alvarado JM, Herrera V. Finger spelling and sign language as alternative codes for reading and writing words for Chilean deaf signers. Am Ann Deaf. 2006;151(3):299-310.

11. Ruegg D, Stefani FM, Cárnio MS. Compreensão de leitura através de interpretação oral e/ou sinalizada de surdos inseridos no contexto de educação especial. Pró-Fono. 1999;11(2):31-7.

12. Associação Brasileira de Empresas de Pesquisa (2008). Critério de Classificação Econômica Brasil. Dados com base no Levantamento Sócio Econômico 2006 e 2007. [citado 2009 Maio 20]. Disponível em:http://www.abep.org/codigosguias/CCEB2008-Base2006e2007.pdf.

13. Pickersgill M. Bilingualism - Current policy and practices. In: Gregory S, Knight P, McCracken W, Powers S, Watson L, editors. Issues in deaf education. London: David Fulton Publishers; 1998. p.88-97.

14. Hoffman PR. Phonological intervention within storybook reading. Top Lang Disord. 1997;17(2):69-88.

15. Eaton JH, Collis GM, Lewis VA. Evaluative explanations in children's narratives of a video sequence without dialogue. J Child Lang. 1999;26(3):699-720.

16. Maloch B. Scaffolding student talk: one teacher's role in literature discussion groups. Read Res Q.2002;37(1):94-112.

17. Fontes MJO, Cardoso-Martins C. Efeitos da leitura de histórias no desenvolvimento da linguagem de crianças de nível sócio-econômico baixo. Psicol Reflex Crit. 2004;17(1):83-94. 
18. Plessow-Wolfson S, Epstein F. The experience of story reading: deaf children and hearing mothers' interactions at story time. Am Ann Deaf. 2005;150(4):369-78.

19. Castillo HV. A leitura de textos literários vs textos científicos por leitores incipientes. In: Witter GP, organizador. Leitura: textos e pesquisas. Campinas: Alínea; 1999.

20. Boruchovitch E. Estratégias de aprendizagem e desempenho escolar: considerações para a prática educacional. Psicol Reflex Crit. 1999;12(2):361-76.
21. Gonçalo SF. Perfil da produção escrita e da trajetória escolar de alunos surdos de ensino médio [dissertação]. São Paulo: Faculdade de Educação da Universidade de São Paulo; 2004.

22. Anaya Nieto D. Efectos del resumen sobre la mejora de la metacomprensión, de la comprensión lectora y del rendimiento académico. Rev Educ. 2005;(337):281-94. 